\title{
Vulval Involvement in Acquired Immunodeficiency Syndrome-Associated Disseminated Histoplasmosis
}

\section{Ramdial PK ${ }^{1 *}$, Sing $\mathbf{Y}^{1}$, Ramburan $\mathrm{A}^{1}$, Nargan $\mathrm{K}^{2}$, Singh $\mathrm{B}^{3}$, Bagratee $\mathrm{JS}^{4}$ and Calonje $\mathrm{E}^{5}$}

${ }^{1}$ Department of Anatomical Pathology, National Health Laboratory Service \& School of Laboratory Medicine and Medical Sciences, University of KwaZulu-Natal, Durban, KwaZulu-Natal, South Africa

${ }^{2}$ KwaZulu-Natal Research Institute for Tuberculosis and HIV, Durban, KwaZulu-Natal, South Africa

${ }^{3}$ Department of General Surgery, Nelson R Mandela School of Medicine, University of KwaZulu-Natal, KwaZulu-Natal, South Africa

${ }^{4}$ Department of Obstetrics and Gynaecology, Nelson R Mandela School of Medicine, University of KwaZulu-Natal, KwaZulu-Natal, South Africa

${ }^{5}$ Department of Dermatopathology, St John's Institute of Dermatology, St Thomas's Hospital, London, UK

\begin{abstract}
Background: Female genital tract, including vulval, histoplasmosis is reported rarely despite an increased propensity for cutaneous involvement by disseminated histoplasmosis (DH), even in patients with acquired immunodeficiency syndrome (AIDS).
\end{abstract}

Methods: Sixteen year retrospective study investigating vulval involvement by histoplasmosis.

Results: Of 239 patients with $\mathrm{DH}, 6$ had vulval involvement and were confirmed to have HIV infection and AIDS. Seventeen biopsies ( 9 vulval, 8 extravulval) from these 6 patients form the study cohort. Patients 1 to 4 had simultaneous vulval (5) and extravulval (5) cutaneous biopsies. Eight cutaneous biopsies demonstrated diffuse dermal infiltration by histiocytes containing budding yeasts of $H$. capsulatum variant capsulatum (HCVC). A single thigh lesion demonstrated diffuse dermal karyorrhexis and myriad extracellular HCVC and a lymph node were diffusely effaced by histiocytes containing HCVC. Patient 5 had concomitant, co-lesional disseminated Kaposi sarcoma and HCVC infection. Patient 6 had 2 initial biopsies that demonstrated $H$. capsulatum variant duboisii (HCVD). Three biopsies of persistent facial and vulval plaques and a vulval ulcer, despite amphotericin treatment, confirmed HCVD, Cytomegalovirus and HCVD and Herpes simplex virus infection in each of the persistent lesions, respectively. Patients 2, 3 and 4 died before treatment was commenced. Patient 5 was lost to follow-up and did not receive any treatment. Patient 1 had resolution of DH following treatment with itraconazole. Persistent cutaneous lesions (Patient 6) healed with aciclovir and ganciclovir but uterine cervical squamous carcinoma was diagnosed 6 months later.

Conclusion: Vulval involvement by histoplasmosis shares overlapping clinical features with many infections and tumors. Vulval biopsies are pivotal for diagnosis and allied therapeutic monitoring, particularly in the context of AIDSassociated co-morbid pathology.

Keywords: Vulva; Histoplasmosis; HIV; AIDS; H. capsulatum

\section{Introduction}

Human histoplasmosis is caused by 2 subspecies of Histoplasma capsulatum, $H$. capsulatum variant capsulatum (HCVC) that causes classical histoplasmosis and H. capsulatum variant duboisii (HCVD) that causes African histoplasmosis [1]. The former is a deep mycosis that exists worldwide but is endemic to regions in the Western Hemisphere including the central United States, Latin American and Caribbean countries [2-4]. Whilst $90-95 \%$ of new infections are asymptomatic, the rest present with symptomatic acute pulmonary, disseminated and chronic pulmonary forms of the disease [5]. Disseminated histoplasmosis (DH), an acquired immunodeficiency syndrome (AIDS)-defining disease [6] that mainly involves the liver, spleen, lymph nodes and bone marrow [7], was documented rarely in the preAIDS era. The mucocutaneous manifestations of $\mathrm{DH}$ are reported in $10-25 \%$ of patients in the USA and in up to $80 \%$ of patients in Latin America [8]. In contrast to the relatively more common reports of genitourinary histoplasmosis in men [9], histoplasmosis of the female genital tract is reported rarely (Table 1) [9-15], even in epidemic regions for histoplasmosis or acquired immunodeficiency syndrome (AIDS). Increased propensity for cutaneous involvement in $\mathrm{DH}$ is also reported $[2,7,8]$, but to date, vulval involvement by HCVC in patients with AIDS has been documented only once in the English-language literature [13]. Furthermore, although cutaneous involvement is a characteristic of African histoplasmosis [16], cutaneous vulval infection by HCVD is unreported to date.
In reporting 6 patients with disseminated, including vulval, histoplasmosis that served as the sentinel of HIV infection and AIDS, we describe the histomorphological spectrum of vulval histoplasmosis (VH), and emphasize clinically unrecognized histoplasmosis as a cause of the vulval lesions. Additionally, we document the hitherto unrecorded impact of vulval co-infections as a cause of a suboptimal response to antifungal therapy, vulval co-lesional occurrence of histoplasmosis and Kaposi sarcoma, and cutaneous vulval involvement by HCVD.

\section{Materials and Methods}

The database of the Department of Anatomical Pathology, University of KwaZulu-Natal and the National Health Laboratory Service, Durban South Africa was accessed to identify all vulval biopsies that were

*Corresponding author: PK Ramdial, Department of Anatomical Pathology, Leve 3, Laboratory Building, Inkosi Albert Luthuli Central Hospital, 800 Vusi Mzimela Road, Mayville, 4058, KwaZulu-Natal, South Africa, Tel: +27 (0)31 2402693; Fax: + 27 (0)31 2402610; E-mail: ramdialpk@gmail.com

Received September 24, 2016; Accepted November 03, 2016; Published November 10, 2016

Citation: Ramdial PK, Sing Y, Ramburan A, Nargan K, Singh B, et al. (2016) Vulval Involvement in Acquired Immunodeficiency Syndrome-Associated Disseminated Histoplasmosis. J AIDS Clin Res 7: 632. doi: 10.4172/2155-6113.1000632

Copyright: (C) 2016 Ramdial PK, et al. This is an open-access article distributed under the terms of the Creative Commons Attribution License, which permits unrestricted use, distribution, and reproduction in any medium, provided the original author and source are credited. 


\begin{tabular}{|c|c|c|c|c|c|c|c|c|}
\hline \multirow[t]{2}{*}{ Year } & \multirow[t]{2}{*}{ Age } & \multirow[t]{2}{*}{ Presentation } & \multicolumn{2}{|c|}{ Genital Histoplasmosis } & \multirow[t]{2}{*}{ E-GH } & \multirow[t]{2}{*}{ S/IIIness } & \multirow[t]{2}{*}{ Treatment } & \multirow[t]{2}{*}{ Outcome } \\
\hline & & & Site & CM & & & & \\
\hline 1959 [10] & 28 & Postpartum fever & LM & ulcers & $\begin{array}{l}\text { Liver, GI, lungs, } \\
\text { spleen, kidney }\end{array}$ & & IV nystatin & Died \\
\hline 1969 [11] & 86 & PVB & Vagina & Ulcer & joints & nil & AmphotericinB & Resolved \\
\hline $1973[14]$ & 27 & Vulvar ulcers & LMi+LM:R & Ulcers & ND & I-LN & No treatment & Resolved \\
\hline 1997 [15] & 63 & Dysuria, PVB & Vagina, LMi:R+L, clitoris & Ulcers & ND & DM & Ketoconazole & Resolved \\
\hline 2000 [12] & 32 & Irregular menses & Ovary (L) & Mass & ND & SLE, C-CF & Ketoconazole & Resolved \\
\hline $2010[9]$ & 42 & $\mathrm{CS}$, rash, PVB & Cervix & Necrotic lesions & BM/skin & AIDS & LAB/itraconazole & ND \\
\hline 2013 [13] & 50 & LM pain, dysuria & LM (L), vagina & Ulcers & ND & AIDS, I-LN & LAB, HAART & $\mathrm{NCH}$ \\
\hline
\end{tabular}

Key: Age: Patient Age in Years; AIDS: Acquired Immunodeficiency Syndrome; BM: Bone Marrow; C-CF: Colocecal Fistula; CM: Clinical Morphology; CS: Non-specific Constitutional Symptoms (fatigue, fever, abdominal pain), DM: Diabetes Mellitus; E-GH: Extra-genital Histoplasmosis; Gl: Gastro-Intestinal Tract; HAART: Highly Active Anti-Retroviral Therapy; I-LN: Inguinal Lymphadenopathy; IV: Intravenous; L: Left; LAB: Liposomal Amphotericin B; LMi: Labium Minus; LM: Labium Majus; NCH: Near Complete Healing; PVB: Per Vaginal Bleeding; ND: Not Documented; R: Right; SLE: Systemic Lupus Erythematosus; S/lliness: Systemic Illnesses; Year: Year of Publication

Table 1: Literature review of histoplasmosis of the female genital tract [9-15].

\begin{tabular}{|c|c|c|c|c|c|c|c|c|c|}
\hline \multirow[t]{2}{*}{ No. } & \multirow[t]{2}{*}{ A/CD4 } & \multirow[t]{2}{*}{ SI } & \multicolumn{3}{|c|}{ Extra-vulval lesions } & \multirow{2}{*}{$\begin{array}{l}\text { Vulval lesions } \\
\text { CM(Size):Site }\end{array}$} & \multirow[b]{2}{*}{ Diagnosis } & \multirow[b]{2}{*}{ Histo } & \multirow[t]{2}{*}{ Outcome } \\
\hline & & & CM/(Biopsy Site) & D/DD & Histo & & & & \\
\hline 1 & $35 / 16$ & PTB & D-*P/N/U (Arm) & TB & $\mathrm{DHI}$ & S-*PI $(2 \mathrm{~cm}):^{*} \mathrm{R}$ LM: & TB/Cancer & $\mathrm{DHI}$ & $\operatorname{Re}$ \\
\hline 2 & $30 / 55$ & Past PTB & $\begin{array}{l}\text { D-*P/N (Leg) } \\
{ }^{*} \text { Inguinal LN }\end{array}$ & TB/NHL & $\mathrm{DHI} / \mathrm{DHI}$ & $\begin{array}{l}M-{ }^{*} U(1.5 \mathrm{~cm}):{ }^{*} L \mathrm{LM} \\
M-U(0.5 \mathrm{~cm}): \mathrm{R}+\mathrm{L} \text { LMi: }\end{array}$ & $\mathrm{GI}$ & $\mathrm{DHI}$ & DWOT \\
\hline 3 & $45 / 34$ & ND & S-*N (Thigh) & Cancer/TB & DK & $\left.M-{ }^{*} \mathrm{PI}(2 \mathrm{~cm})\right)^{*} U(2 \mathrm{~cm}){ }^{*} \mathrm{R}$ LM & Syphilis/TB & $\mathrm{DHI}$ & DWOT \\
\hline 4 & $22 / 3$ & ND & D-*P/N/U (Neck) & TB & DHI/DK & M-*P/PI/N:R+*L LM & Cancer/TB & $\mathrm{DHI}$ & DWOT \\
\hline 5 & $26 / 46$ & ND & $\mathrm{D}-{ }^{*} \mathrm{P} /{ }^{*} \mathrm{~N} / \mathrm{U}$ (back) & $\mathrm{NHL}$ & $\mathrm{KS} / \mathrm{DHI}$ & M-*P/N (haemorrhagic):* R LM & $\mathrm{KS} / \mathrm{NHL}$ & $\mathrm{KS} / \mathrm{DHI}$ & LTFU \\
\hline \multirow[t]{2}{*}{6} & \multirow[t]{2}{*}{$38 / 42$} & \multirow{2}{*}{$\begin{array}{l}\text { Cervix } \\
\text { SCC }\end{array}$} & D-*P/PI/N (Neck) & TB & DHI & $M-{ }^{*} U(5 \mathrm{~mm}): R L M+{ }^{*}$ LLM & TB/GI & DHI & \multirow[t]{2}{*}{ Re/LTFU } \\
\hline & & & S-\#PI (Face) & $\mathrm{TB} / \mathrm{Ca}$ & DHI & M- ${ }^{*} \cup(5 \mathrm{~mm})+\# P I(3 \mathrm{~cm}):{ }^{*} R$ LM & Cancer/KS & $\mathrm{DHI}$ & \\
\hline
\end{tabular}

Key: A: Age, CD4:CD4 T-Lymphocytes in $\mathrm{mm}^{3}$, CM: Clinical Morphology; D: Disseminated; D/DD: Clinical Diagnosis/Differential Diagnosis; DHI: Diffuse Histiocytic in Filtrate with Intracytoplasmic H. capsulatum; DK: Diffuse Karyorrhexis with mainly Extracellular $H$. capsulatum; DWOT: Died Without Treatment, GI: Granuloma Inguinale; Histo: Histomorphological Features; KS: Kaposi Sarcoma; L: left, LM: Labium Majus, LMi: Labium Minus, LN: Lymph Node, LTFU: Lost to Follow-up, M: Multiple; N: Nodules; ND: Not Documented; NHL: Non-Hodgkin Lymphoma; No: Patient Number; P: Papules; PI: Plaques; PTB: Pulmonary Tuberculosis; R: Right, Re: Resolved; S: Single; SCC: Invasive Squamous Cell Carcinoma; SI: Systemic Illness; TB: Tuberculosis, U: Ulcers; *: Morphology of initial biopsied lesion/s; \#: Patient on treatment at time of second set of biopsies

Table 2: Summary of clinicopathological findings.

diagnosed and coded as histoplasmosis from 1 January 1998 to 31 December 2014; using the SNOMED word and code search engines. Clinical data, including patient age, distribution and morphology of the cutaneous lesion/s, the presence of systemic disease, HIV status, CD4 count, response to treatment and disease outcome, was extracted from departmental and patient records. Stored slides or recut and stained hematoxylin and eosin (H\&E), periodic acid Schiff (PAS), Southgate mucicarmine, Fontana-Masson and Gomori Grocott methenamine silver (GGMS) stained sections were re-assessed. Cytomegalovirus (CMV) (clone: CCH2, 1:400 dilution, Dakopatts, Glostrup, Denmark), Herpes simplex virus (HSV) (polyclonal, 1:300 dilution, Dakopatts, Glostrup, Denmark) and Human herpesvirus 8-latent nuclear antigen-1 (HHV8-LNA-1) (clone: 13B10, Novocastra, Newcastle-upon-Tyne, United Kingdom) immunohistochemistry were undertaken on vulval biopsies from 1 patient each, using a microwave-based, heat-assisted antigen retrieval technique as per standard methodology. Both stains employed hematoxylin as the counterstain. The Novolink polymer detection system (Leica Microsystems, Newcastle Upon Tyne, United Kingdom) and diaminobenzidine chromogen were employed for antibody visualization.

Molecular investigation was undertaken on a cutaneous facial plaque biopsy from patient 6 (P6). Briefly, DNA was extracted using the QIAamp FFPE DNA extraction kit (Qiagen, Valencia, CA, USA) according to manufacturer's instruction (Qiagen, Valencia, CA, USA). The integrity of the extracted DNA was assessed by polymerase chain reaction (PCR) for a 309-base pair segment of the Beta actin gene [17]. Twenty nanograms of genomic DNA was used in each $25 \mu$ PCR using the
FastStart Taq DNA polymerase PCR kit (Roche Bioscience, Palo Alto, CA, USA) according to manufacturer's instruction (Roche Bioscience, Palo Alto, CA, USA). PCR was performed on a CFX-96 (Bio-Rad, Hercules, CA, USA) thermal cycler. Thereafter, PCR for the $25 S$ rRNA 3'end ETS and $18 S$ rRNA gene of Histoplasma capsulatum was performed using primers; Forward 5'-CTCGAGCATCCGCGGTATC-3' and Reverse 5'-CTGGCAGAATCAACCAGGTAGCTAG-3' (Roche Bioscience, Palo Alto, CA, USA). The 439 base pair product that was generated was subsequently nucleotide sequenced by Inqaba Biotechnical Industries (Hatfield, Pretoria, South Africa).

\section{Results}

\section{General clinical details}

Six of 239 patients in the study period with $\mathrm{DH}$ had vulval involvement and form the patient study cohort. Seventeen incisional cutaneous biopsies, 9 vulval and 7 extravulval and an inguinal lymph node excision (Table 2) that were undertaken for diagnostic purposes, were re-reappraised. The mean patient age was 32.7 (range=22-45) years. HIV testing, following the diagnosis of cutaneous histoplasmosis, confirmed HIV seropositivity in all patients. The CD4 T-lymphocyte count ranged from 3 to $55\left(\right.$ mean=32.7) cells $/ \mathrm{mm}^{3}$. The cutaneous lesions served as the sentinel of DH, HIV infection and AIDS in the study cohort.

\section{Specific clinicopathological features: Patients 1 to 4 (P1 to P4)}

Clinical features: While all patients presented primarily for 
Citation: Ramdial PK, Sing Y, Ramburan A, Nargan K, Singh B, et al. (2016) Vulval Involvement in Acquired Immunodeficiency Syndrome-Associated Disseminated Histoplasmosis. J AIDS Clin Res 7: 632. doi: 10.4172/2155-6113.1000632

Page 3 of 8

management of the cutaneous lesions, all were emaciated and patient 3 also complained of dyspnea. Variably tender, papular, nodular and ulcerative cutaneous lesions were widely disseminated on the scalp, neck, face, trunk, upper and lower limbs, buttock, genital and perineal skin of all patients. Three patients had multiple vulval lesions. Whereas malignancies and infections of varied origin were clinical considerations, histoplasmosis was not (Table 2).

Histopathological features: Two biopsies (patients 1,2 and 4) and 3 biopsies (patient 3 ) were undertaken simultaneously from vulval and extravulval sites (Table 2). All vulval cutaneous biopsies, extravulval cutaneous biopsies from P1, P2 and P4 and the lymph node from P2 demonstrated a diffuse infiltrate of histiocytes with voluminous cytoplasm (Figure 1A) containing round to oval organisms, 2 to $4 \mu \mathrm{m}$ in diameter (Figures $1 \mathrm{~B}$ and $1 \mathrm{C}$ ), with perifungal clear spaces and variable narrow-necked budding, in keeping with HCVC infection. While a solitary cutaneous thigh nodule from P3 demonstrated diffuse pandermal karyorrhexis (Figure 1D) with a myriad extracellular HCVC amidst nuclear debris (Figure 1E) and transfollicular fungal elimination (Figures $1 \mathrm{~F}$ and $1 \mathrm{G}$ ), such karyorrhectic foci were a focal finding in the other vulval and extravulval cutaneous biopsies. Focal non-necrotizing granulomatous inflammation was evident in an extravulval cutaneous biopsy from P2. Scattered lymphocytes and plasma cells were present.
Intravascular circulating monocytes with $\mathrm{HCVC}$ were noted in all biopsies (Figures $2 \mathrm{~A}$ and $2 \mathrm{~B}$ ). Intra-histiocytic fungi were noted within nerves in 1 biopsy each from the labium majus of P1 and P3 (Figure 2C). Biopsies of the vulval plaques from Pland P3 demonstrated pseudoepitheliomatous hyperplasia and transepidermal fungal elimination (Figures 2D and 2E).

Outcome: Culture of extravulval cutaneous lesions was undertaken following histopathological diagnosis; these confirmed HCVC. Fungal yeast forms of HCVC were also confirmed in sputum samples from P3. Patient 1 was treated with amphotericin B intravenously for 3 days and was discharged on itraconazole. At follow-up 4 weeks later, there was dramatic healing of her genital and extragenital cutaneous lesions. Patients 2, 3 and 4 died within the first 3 days of admission without any treatment.

\section{Specific clinicopathological features: Patient 5 (P5)}

Clinical features: P5 had disseminated, slightly hyperpigmented cutaneous lesions (Figure 3A). Because of the varied morphological features and clinical suspicions, 1 vulval and 2 extravulval biopsies were undertaken for histopathological assessment.

Histopathological features: Vulval and extravulval cutaneous
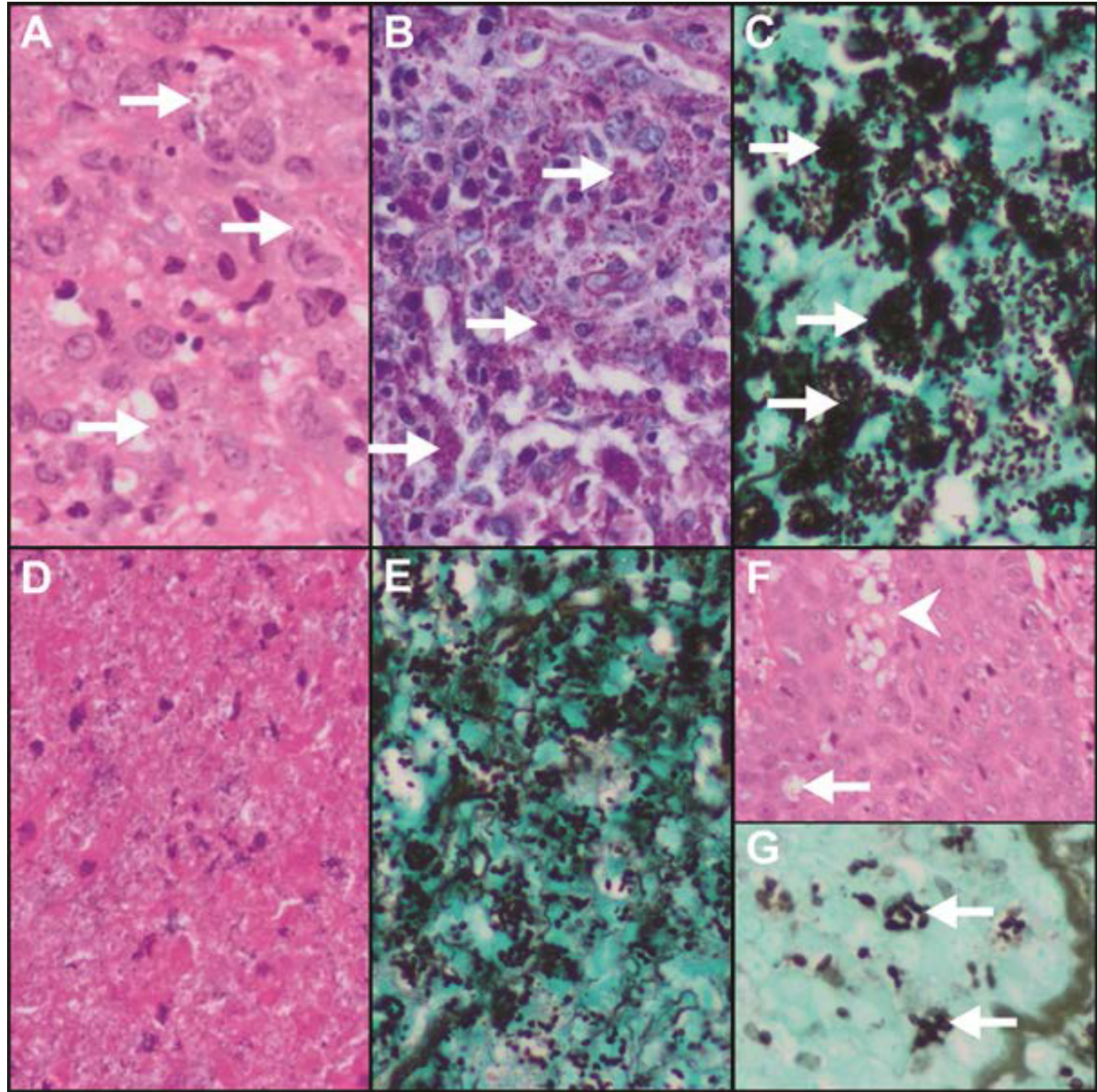

Figure 1: Cutaneous extravulval papular lesion (patient 1) with a diffuse infiltrate of histiocytes parasitized by HCVC [A, arrows (H\&E 480X)], confirmed on PAS [B, arrows $(480 X)]$ and GGMS [C, arrows $(480 X)]$ stains. Cutaneous extravulval thigh nodule (patient 3 ) demonstrating pandermal diffuse karyorrhexis [D $(H \& E$ 480X)] with a myriad extracellular organisms [E (GGMS 120X)] and transfollicular fungal elimination [F, arrow (arrowhead=sebocytes) (H\&E 480X)] highlighted by GGMS staining $[\mathrm{G}$, arrows $(480 X)]$. 
Citation: Ramdial PK, Sing Y, Ramburan A, Nargan K, Singh B, et al. (2016) Vulval Involvement in Acquired Immunodeficiency Syndrome-Associated Disseminated Histoplasmosis. J AIDS Clin Res 7: 632. doi: 10.4172/2155-6113.1000632

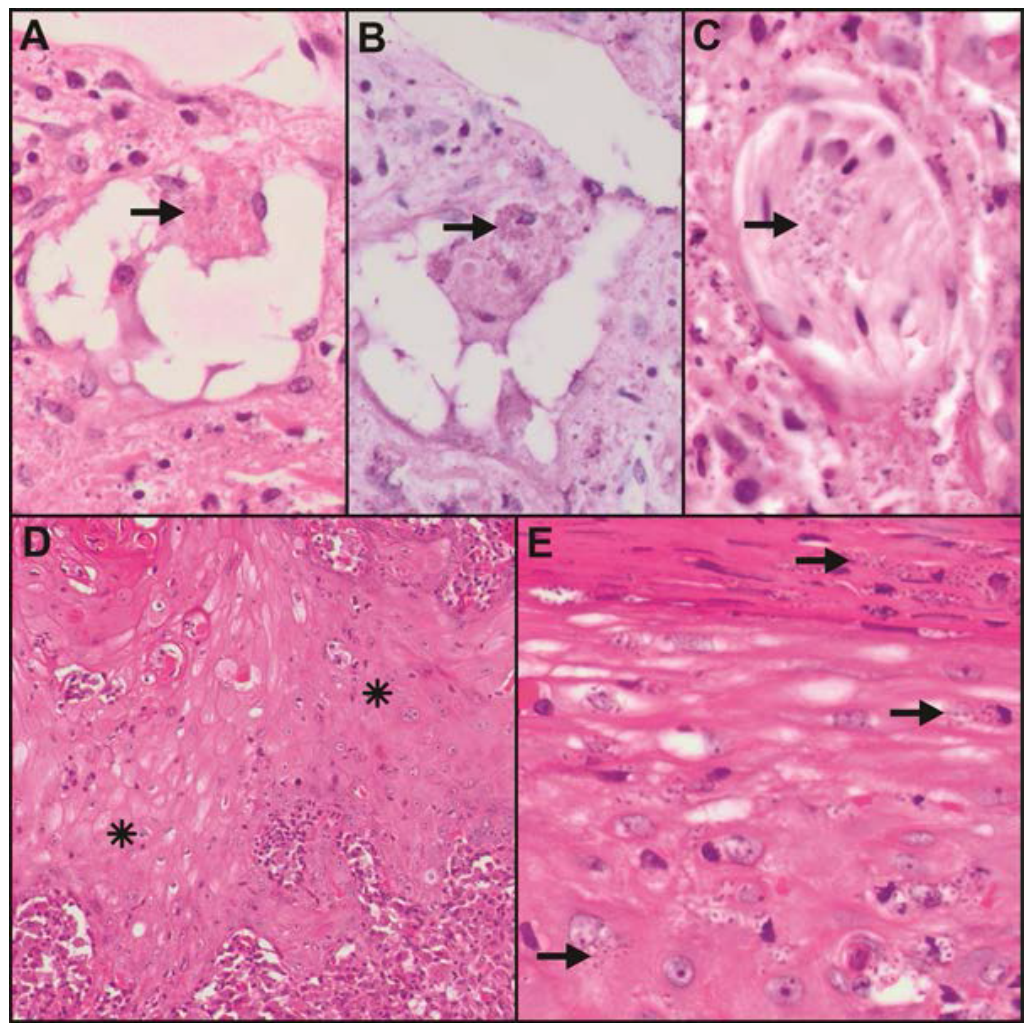

Figure 2: Vulval biopsy (patient 3) with intravascular, intrahistiocytic HCVC [A, arrow (H\&E 240X)] illuminated by PAS staining [B, arrow (240X)], intraneural organisms $[C$, arrow $(H \& E 240 X)]$, pseudoepitheliomatous hyperplasia [D, asterisks $(H \& E, 480 X)]$ and transepidermal fungal elimination $[E$, arrows $(H \& E$ 480X)].

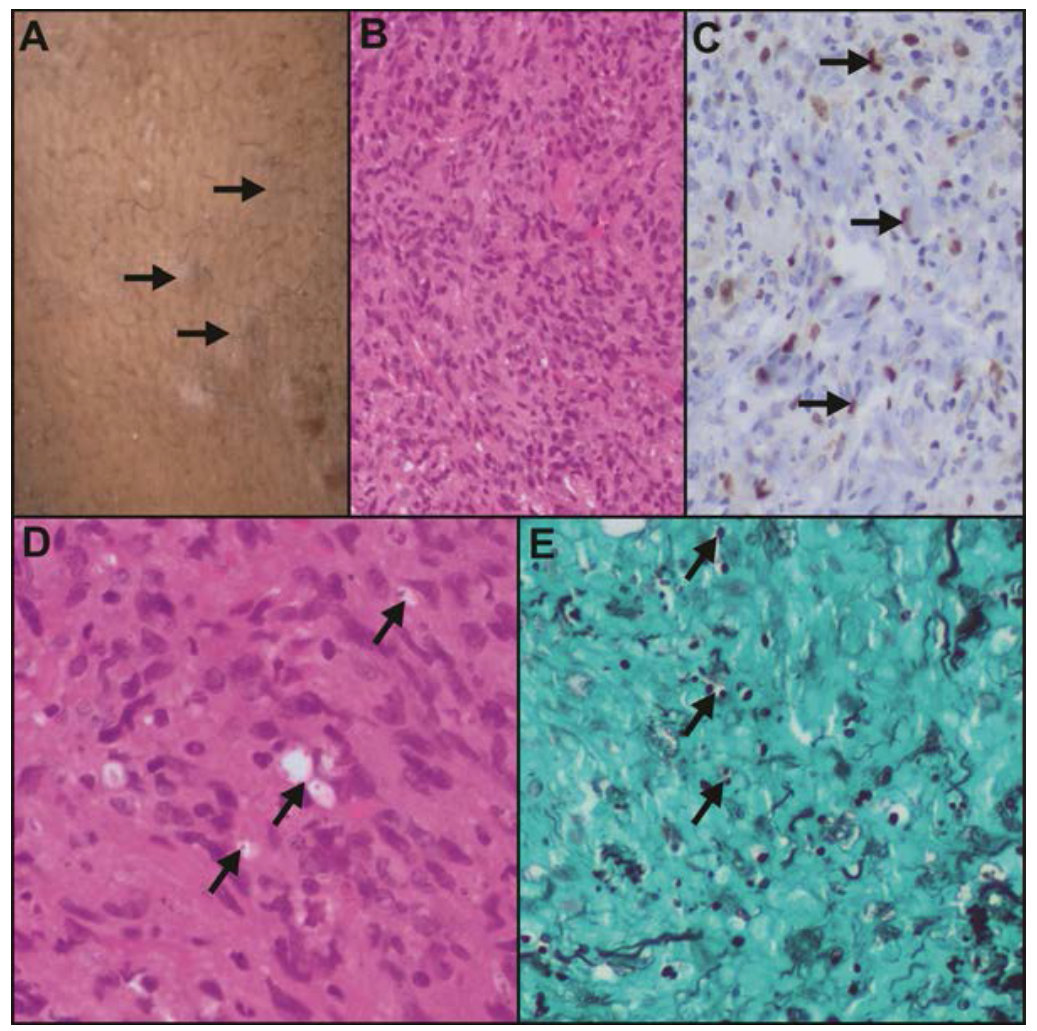

Figure 3: Patient 5 with extravulval Kaposi sarcoma [A, arrows] and vulval Kaposi sarcoma composed of malignant spindle cells [B (H\&E 120X)]. HHV8-LNA-1 immunopositive tumour cells [C, arrows (HHV8-LNA-1 120X)]. Admixed histiocytes with intracytoplasmic fungi [D, arrows (H\&E 240X)], highlighted by GGMS staining [E, arrows]. 
biopsies demonstrated pandermal collagen dissection by spindle cells and capillary-caliber vasculature (Figure 3B). The neoplastic cells were HHV8LNA-1-immunopositive (Figure 3C). Hyaline globules, erythrocyte extravasation, hemosiderin pigment deposition and a lymphoplasmacytic infiltrate were evident. In addition, histiocytes containing organisms with the morphology of HCVC were admixed with the spindle cell component (Figures 3D and 3E). A diagnosis of disseminated co-lesional cutaneous Kaposi sarcoma and histoplasmosis was made.

Outcome: Culture of an extravulval cutaneous lesion, following the histopathological diagnosis, confirmed HCVC. P5 did not return for further follow-up.

\section{Specific clinicopathological features: P6}

Clinical features: Two biopsies from the neck and left labium majus were received initially. Following biopsy diagnosis, P6 received oral antifungal therapy for 7 days. There was variable resolution of most of the cutaneous lesions at her follow-up visit, but she complained of persistent pain and increase in the size of the facial plaque and vulval lesions. Biopsy of the facial and vulval plaques and of the right labium majus ulcer was then undertaken.

Histopathological features: The initial extravulval and vulval cutaneous biopsies from patient 6 (Figures 4A-4C) demonstrated a diffuse infiltrate of large histiocytes and multinucleate giant cells with 8 to $14 \mu \mathrm{m}$ intracytoplasmic yeast forms (Figure 4A) with thick, refractile walls (Figure 4B). GGMS stains highlighted fungal chains, figure-of- 8 and narrow-necked budding forms (Figure 4C). Southgate mucicamine and Fontana-Masson stains were negative. These features were compatible with HCVD and online bioinformatics investigations [18] of the PCR-generated fragment confirmed the same.
Subsequent biopsy of the persistent right labium majus and facial plaques demonstrated variable ulceration and pseudoepitheliomatous hyperplasia with transepidermal fungal elimination, dermal fibrosis and admixed HCVD-laden histiocytic aggregates, scattered neutrophils, lymphocytes and plasma cells. In addition, biopsy of the plaque in the right labium majus also demonstrated intranuclear owl's eye and amphophilic cytoplasmic inclusions of CMV in endothelial cells of thrombosed vasculature, histiocytes, eccrine units and myofibroblasts (Figures 5A and 5B). The superficial shave biopsy of the left labium majus ulcer demonstrated HSV inclusions within the nucleus of keratinocytes, some of which were multinucleate (Figures 5C and 5D).

Outcome: Aciclovir and ganciclovir were added to the patient's therapeutic regimen. Resolution of the skin lesions was confirmed at 6 weeks. Biopsy of a uterine cervical mass 6 months later confirmed an invasive squamous cell carcinoma. She was lost to further follow-up.

\section{Discussion}

DH occurs predominantly in individuals with compromised immunity or with a massive inoculum [12]. Classical histoplasmosis, documented in 1 in 1000 to 1 in 500000 people in the pre-AIDS era, mainly in transplant recipients, in patients on immunosuppressive therapy, with hematologic malignancies, inborn errors of immunity or at the extremes of age $[4,5,12]$, is acquired by inhalation of spores from soil contaminated by bird and bat guano $[4,12]$. Patients with AIDS and low $\mathrm{CD} 4$ counts, however, are at greatest risk for $\mathrm{DH}$, documented as the first presentation of opportunistic infections in $75 \%$ of patients with AIDS [8]. Cutaneous lesions occur in approximately $50 \%$ of AIDS patients with disseminated classical histoplasmosis [3]. In contrast to classical histoplasmosis, the ecological and pathogenetic profile

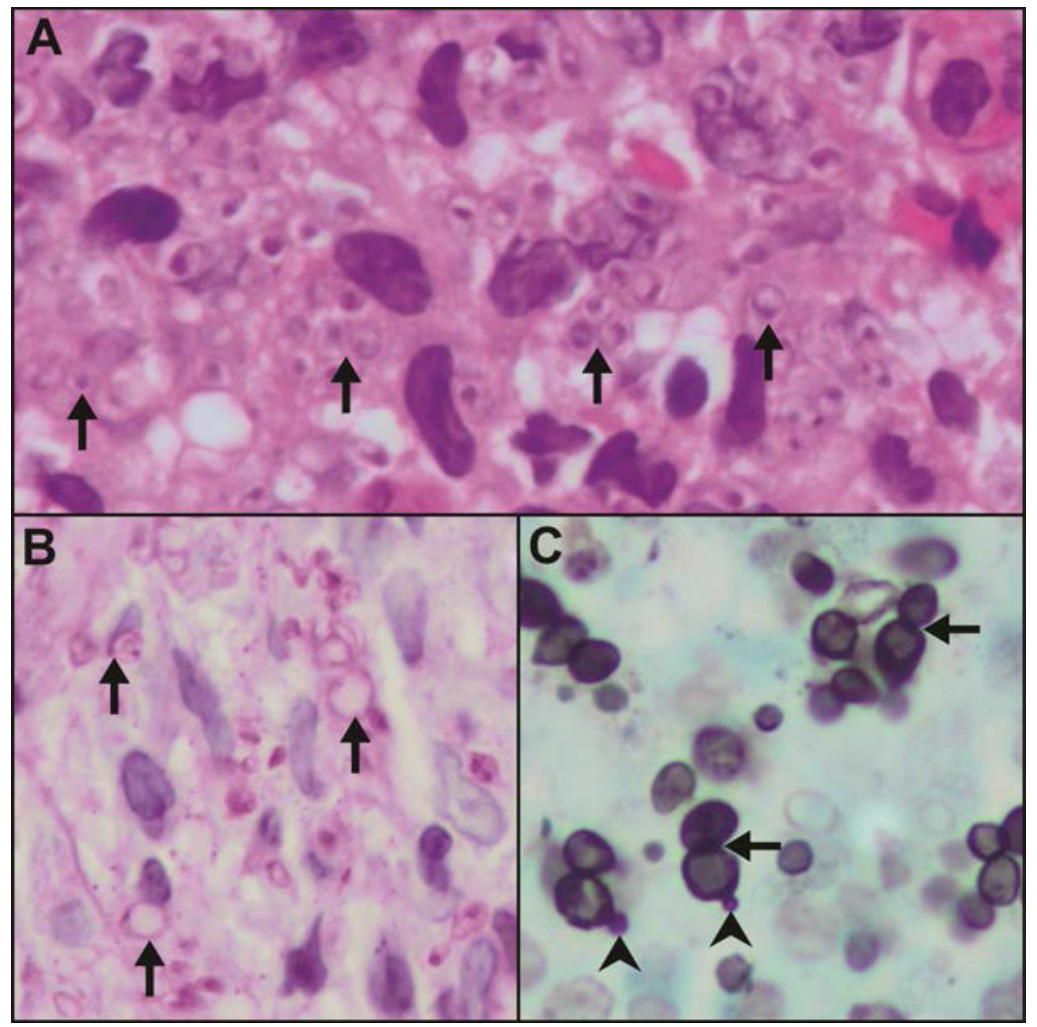

Figure 4: Vulval biopsy from patient 6: Transdermal and subcutaneous HCVD [A, arrows (H\&E)] with prominent capsules [B, arrows (PAS oil immersion 1200X)] and figure-of-8 morphology [C, arrows (GGMS oil immersion 1200X)] and narrow-necked budding [C, arrowheads]. 


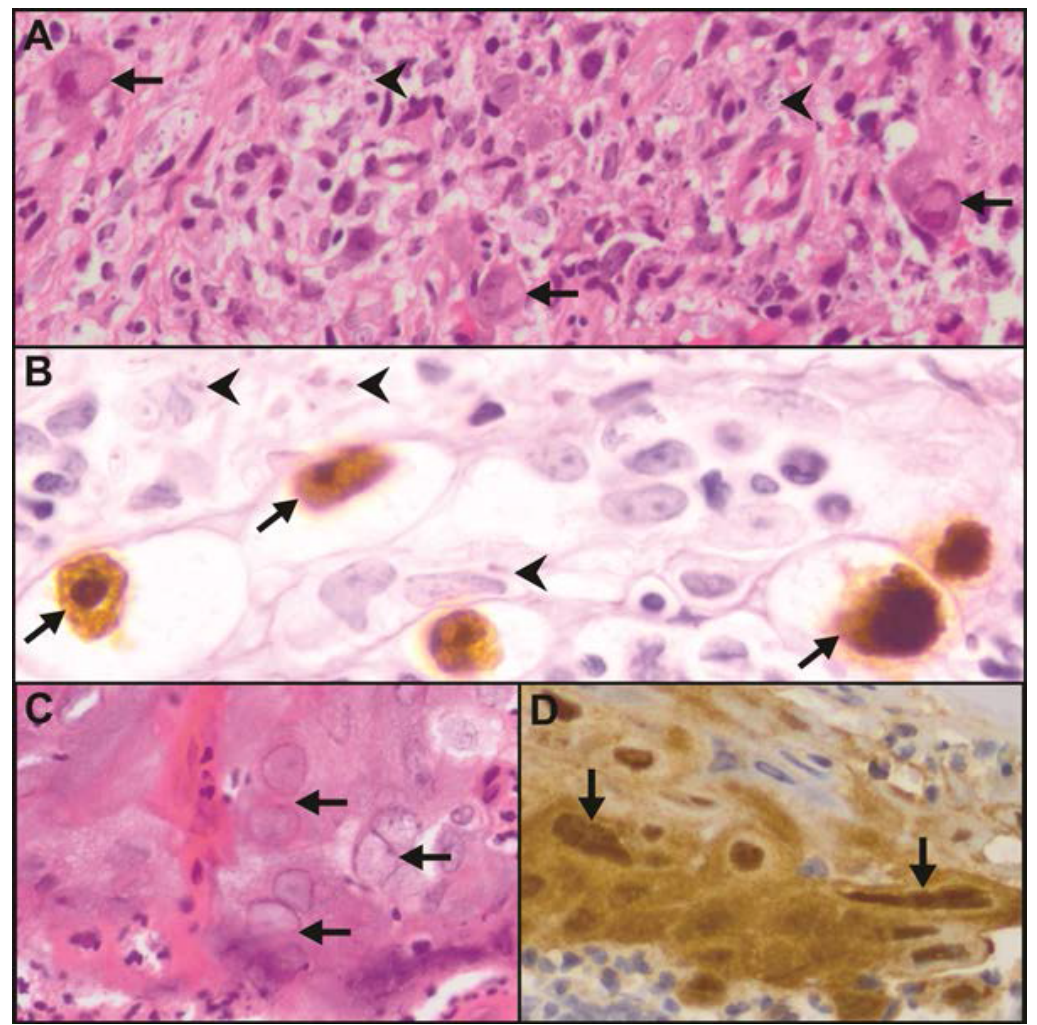

Figure 5: Vulval biopsies from patient 6: CMV inclusions within endothelial cells [A, arrows (H\&E 240X)], confirmed immunohistochemically [B, arrows (CMV oil immersion 1200X)] and surrounding HCVD [B, arrowheads]. Intranuclear HSV inclusions in keratinocytes [C, arrows (H\&E 480X)], confirmed immunohistochemically [D, arrows $(H S V, 480 X)]$

of African histoplasmosis, first described in 1952 [19] and endemic in West and Central Africa and Madagascar, is poorly understood [1]. Classical histoplasmosis is typified by predominant pulmonary involvement while African histoplasmosis is characterized mainly by cutaneous, subcutaneous and osseous disease [19]. The cutaneous lesions, however, comprise a spectrum of manifestations, including papules, pustules, nodules, ulcers, acneiform lesions, verrucous plaques, vesicles, purpura and erythema multiforme-like lesions [1-4,8,19]. Because of the clinical heterogeneity, the definitive diagnosis of classical and African histoplasmosis requires culture and fungal isolation from clinical secretions or biopsy samples [20].

While genital histoplasmosis is not uncommon in males, female genital tract histoplasmosis has been documented rarely in the Englishlanguage literature (Table 1) [9-15]. Of these, 1 patient each had ovarian, vulval, vaginal and cervical histoplasmosis and 2 had vulvovaginal histoplasmosis. Two of the 6 reported patients with cervical and vulvovaginal histoplasmosis were HIV-positive. It is surprising that vulval cutaneous involvement in patients with $\mathrm{DH}$ is documented rarely in patients with AIDS and DH. Whether this represents a true rarity or an under-reporting phenomenon is uncertain. It is possible, however, that when patients with $\mathrm{DH}$ and lesions in exposed skin are in an ill state, little attention is paid to lesions in unexposed sites. Hence, the rarity of vulval disease may be a function of non-reporting rather than true non-occurrence. In the present study, while all patients sought medical attention for disseminated cutaneous lesions, the vulval lesions were biopsied because the patients complained of pain therein, a poor response to therapy or to exclude potential dual co-morbid pathology in genital and extragenital locations. The latter indication arose because the vulval lesions were not distinctive clinically, and the clinical suspicions included infections such as tuberculosis, granuloma inguinale and syphilis and malignancies that included squamous cell carcinoma and Kaposi sarcoma (Table 2). The vulval lesion of patient 5 was biopsied to confirm the clinical suspicion of Kaposi sarcoma. Concomitant co-lesional Kaposi sarcoma and histoplasmosis has been diagnosed once in the literature [21], but such co-occurrence is unreported in the vulva. Less attention was paid to the genital disease by $\mathrm{P} 6$ and the attending clinician at the initial visit. The vulval lesions were re-biopsied because of increasing lesional pain and the suboptimal response of the vulval ulcers and plaque to initial antifungal therapy. Hence, vulval biopsies were important to distinguish histoplasmosis from other vulval infections and tumors and to confirm the co-existence of co-morbid infective and neoplastic diseases.

The well-known histopathological patterns of DH include diffuse dermal histiocytic infiltration, dermal karyorrhexis and necrotizing and non-necrotizing granulomatous inflammation [2-5,8]. More recently, lichenoid, nodular pseudomyxoid, pyogenic granulomalike, perifollicular, superficial, mid and deep dermatitic, focal nodular dermatitic and histiocytic lobular panniculitic patterns have been documented in AIDS-associated DH [22]. The clinical spectrum of cutaneous $\mathrm{VH}$ in the present study was similar to that commonly documented in the literature, including ulcers, papules, papulonodules and plaques $[4,5,8]$. The cutaneous histopathological spectrum was limited to diffuse, pandermal histiocytic infiltration, diffuse, pandermal karyorrhexis and focal non-necrotizing granulomatous inflammation. Histoplasmosis was not suspected as a cause of the vulval disease clinically but a suspicion of sexually transmitted diseases and 
malignancies prompted vulval biopsies. Whether the painful nature of the genital lesions was a function of the rich neural supply of the vulval/ perineal region or whether it is a clinical reflection of $H$. capsulatum invasion of the nerve plexus is uncertain, as is the contributory role of the viral co-infections in the pathogenesis of pain. Notwithstanding this uncertainty, it is important to recognize that the painful nature of the genital lesions prompted vulval biopsies and diagnosis of $\mathrm{VH}$ in all patients, in addition to co-lesional, co-morbid diseases in 2 patients. In 4 patients $\mathrm{DH}$ represented advanced fatal disease. While long term follow-up was unavailable for 2 patients, it is noteworthy that despite their profound immunocompromize, their cutaneous lesions responded to appropriate, timely therapy.

Because of the non-specific nature of the cutaneous disease, histopathological, mycological, serological and molecular tests are helpful in definitive diagnosis $[23,24]$. While fungal culture has been the gold standard for the diagnosis of histoplasmosis, the diagnostic technique is disadvantaged by the slow growth characteristics of $H$. capsulatum and the challenges of laboratory-based temperaturedependent mycelium-to-yeast transformation [24]. H. capsulatum antigen tests may be positive in $92 \%$ and $83 \%$ of urine and serum samples from patients with AIDS, respectively, but fungal cross reactivity and immunocompromize-related negativity may produce incorrect results $[19,24]$. Histopathological assessment not only facilitates rapid diagnosis of histoplasmosis but also enables confirmation of co-lesional co-infections, as was present in 2 vulval biopsies from patient 4 . As in P6, confirmation of histoplasmosis is also possible from wax-embedded tissue using PCR [24].

The complete careful appraisal of genital tract lesions with a granulomatous, diffuse histiocytic and karyorrhectic background with optimally undertaken special stains for infections, are invaluable in overcoming the mimicry that is associated with the infections that may involve the vulva. These special stains include Ziehl Neelsen, FiteFaraco, PAS, GGMS, Warthin-Starry and Giemsa. As evidenced in this report, a range of infective and non-infective diseases may mimic the histopathological spectrum of $\mathrm{VH}$. The recognition of the 2-4 $\mu \mathrm{m}$ budding yeast forms is the gold standard for diagnosis of HCVC $[1,4,5]$. The presence of granulomas with or without necrosis may mimic secondary or tertiary syphilis, tuberculosis, cryptococcosis and leishmaniasis. Secondary syphilis may be accompanied by epidermal alterations including a lichenoid or psoriasiform phenotype and endothelial cell swelling. When present, a dense plasma cell infiltrate facilitates the diagnosis [25]. The recognition of spirochetes using silver stains and T. pallidum by immunohistochemistry is helpful for microscopic confirmation, but partially treated syphilis may pose diagnostic challenges. The role of PCR for T. pallidum confirmation in formalin-fixed paraffin-embedded tissue and correlation with treponemal serology cannot be overvalued in such settings. While $C$. neoformans is uncommon in the female genital tract, the identification of $C$. neoformans may be a challenge in paucifungal granulomatous lesions and when capsule-deficient $C$. neoformans predominates. In the former, use of mucicarmine stains will be helpful and in the latter, Fontana Masson staining will differentiate C. neoformans from $H$. capsulatum [26]. The smaller size of leishmanial organisms and the identification of the kinetoplast on Giemsa-stained sections distinguish leismaniasis from histoplasmosis [25,27]. Fite-Faraco stains may assist the identification of acid fast bacilli in tuberculoid leprosy [28], especially in nerves, but mycobacterial PCR studies may be necessary to demonstrate a definitive $M$. leprae origin.

Biopsies with a diffuse histiocytic infiltrate containing "parasitized"
H. capsulatum mimic leishmaniasis, lepromatous leprosy, penicilliosis and granuloma inguinale. The first 2 infective agents are smaller than HCVC and the Giemsa and Fite-Faraco special stains are invaluable in confirming each one, respectively $[4,25]$. The presence of septate yeasts is useful in differentiation of $P$. marneffei from HCVC yeasts with narrow-necked budding [29] especially as these infective agents have overlapping clinical manifestations, laboratory findings and chest radiographic abnormalities [30]. While Donovan bodies of granuloma inguinale may mimic HCVC, compelling clues to granuloma inguinale include the plasma cell background with microabscesses, absence of budding organisms and smaller size of the causative agent, Klebsiella granulomatis [25].

Because of the larger size and shape characteristics of HCVD, cutaneous cryptococcosis, penicilliosis and blastomycosis may pose diagnostic challenges [31]. C. neoformans may also grow in chains but the carminophilous capsule sets it apart from HCVD $[25,26]$. P. marneffei demonstrates distinct septation and $B$. dermatitidis has multiple nuclei and broad-based budding [31]. Lastly, biopsies with karryorrhectic foci may mimic non-infective, inflammatory conditions including lupus erythematosus, Sweet syndrome and vasculitis, but special fungal stains are diagnostic. While the clinical features of these patients may be helpful, careful attention to the variation in size of nuclear fragments and the presence of fungal forms with distinctive, well-defined contours on silver stains underpins the diagnosis of an infective process.

In conclusion, vulval involvement as part of $\mathrm{DH}$ is eminently treatable. The clinical morphological features of vulval involvement by $\mathrm{DH}$ are not distinctive. $\mathrm{VH}$ shares overlapping clinical features with a range of infections and tumors. Hence, biopsy of vulval lesions is pivotal, not only for diagnosis and monitoring of a therapeutic response, but also, as noted in this study, for the recognition of co-infective pathology and malignancy. The spectrum of cutaneous disease, including vulval involvement, served not only as the initial clue to $\mathrm{DH}$, but also as a sentinel of HIV infection, AIDS, Kaposi sarcoma and CMV and HSV co-infections in the study patients.

\section{Acknowledgement}

We thank Mrs. M Moodley for manuscript word processing, formatting and assistance with literature access and Mr. Dinesh Sookhdeo for patient data collation.

\section{Declaration}

Permission to undertake this study was obtained from the Biomedical Research Ethics Committee of the University of KwaZulu-Natal (Reference Number BCA535/16).

\section{References}

1. Loulergue P, Bastides F, Baudouin V, Chandenier J, Mariani-Kurkdjian P, et al. (2007) Literature review and case histories of Histoplasma capsulatum var duboisii infections in HIV-infected patients. Emerg Infect Dis 13: 1647-1652.

2. Bonifaz A, Cansela R, Novales J, de Oca GM, Navarrete G, et al. (2000) Cutaneous histoplasmosis associated with acquired immunodeficiency syndrome (AIDS). Int J Dermatol 39: 35-38.

3. Friedman A, Solomon G, Segal-Maurer S, Pereira F (2008) Sudden onset of verrucous plaques to the face and trunk: $A$ case of reactivation cutaneous histoplasmosis in the setting of HIV. Dermatol Online J 14: 12.

4. Ramdial PK, Mosam A, Dlova NC, Satar B, Aboobaker J, et al. (2002) Disseminated cutaneous histoplasmosis in patients infected with human immunodeficiency virus. J Cutan Pathol 29: 215-225.

5. Chandler FW, Watts JC (1997) Histoplasmosis capsulati. Pathology of infectious diseases. Appleton \& Lange, Stamford, Connecticut, USA 1007-1015.

6. Centers for Disease Control (1985) Revision of the case definition of acquired 
Citation: Ramdial PK, Sing Y, Ramburan A, Nargan K, Singh B, et al. (2016) Vulval Involvement in Acquired Immunodeficiency Syndrome-Associated Disseminated Histoplasmosis. J AIDS Clin Res 7: 632. doi: 10.4172/2155-6113.1000632

immunodeficiency syndrome for national reporting-United States. MMWR Morb Mortal Wkly Rep 34: 373-375.

7. Dijkstra JW (1989) Histoplasmosis. Dermatol Clin 7: 251-258.

8. Bonifaz A, Chang P, Moreno K, Fernández-Fernández V, de Oca GM, et al. (2009) Disseminated cutaneous histoplasmosis in acquired immunodeficiency syndrome: Report of 23 cases. Clin Exp Dermatol 34: 481-486.

9. Cantor AG, John J, Brown B (2010) Metrorrhagia in a human immunodeficiency virus positive woman caused by cervical histoplasmosis. Am J Med Sci 339: 285-287.

10. Conrad FG, Saslaw S, Atwell RJ (1959) The protean manifestations of histoplasmosis as illustrated in twenty-three cases. Arch Intern Med 104: 692709 .

11. Gass M, Kobayashi GS (1969) Histoplasmosis. An illustrative case with unusual vaginal and joint involvement. Arch Dermatol 100: 724-727.

12. Isotalo PA, McCarthy AE, Eidus L (2000) Ovarian histoplasmosis in systemic lupus erythematosus. Pathology 32: 139-141.

13. Shine MM, Shah MM, Hanna CA, Bevis KS (2013) Vulvar histoplasmosis as a rare cause of genital ulceration. Obstet Gynecol 122: 449-452.

14. Sills M, Schwartz A, Weg JG (1973) Conjugal histoplasmosis. A consequence of progressive dissemination in the index case after steroid therapy. Ann Int Med 79: 221-224.

15. Smith MB, Schnadig VJ, Zaharopoulos P, Van Hook C (1997) Disseminated Histoplasma capsulatum infection presenting as genital ulcerations. Obstet Gynecol 89: 842-844.

16. Gonçalves D, Ferraz C, Vaz L (2013) Posaconazole as rescue therapy in African histoplasmosis. Braz J Infect Dis 17: 102-105.

17. Richter E, Schlüter C, Duchrow M, Hahn M, Rüsch-Gerdes S, et al. (1995) An improved method for the species-specific assessment of mycobacteria in routinely formalin-fixed and paraffin-embedded tissues. J Pathol 175: 85-92.

18. Genbank (2016) NCBI.

19. Tsiodras S, Drogari-Apiranthitou M, Pilichos K, Leventakos K, Kelesidis T, et al. (2012) An unusual cutaneous tumor: African histoplasmosis following mud baths: Case report and review. Am J Trop Med Hyg 86: 261-263.
20. Garcia-Guiñon A, Torres-Rodríguez JM, Ndidongarte DT, Cortadellas F, Labrín L (2009) Disseminated histoplasmosis by Histoplasma capsulatum var duboisi in a paediatric patient from the Chad Republic, Africa. Eur J Clin Microbiol Infect Dis 28: 697-699.

21. Cole MC, Cohen PR, Satra KH, Grossman ME (1992) The concurrent presence of systemic pathogens and cutaneous Kaposi's sarcoma in the same lesion: Histoplasma capsulatum and Kaposi's sarcoma coexisting in a single skin lesion in a patient with AIDS. J Am Acad Dermatol 26: 285-287.

22. Ollague Sierra JE, Ollague Torres JM (2013) New clinical and histological patterns of acute disseminated histoplasmosis in human immunodeficiency virus-positive patients with acquired immunodeficiency syndrome. Am J Dermatopathol 35: 205-212.

23. Mehta V, De A, Balachandran C, Monga $P$ (2009) Mucocutaneous histoplasmosis in HIV with an atypical ecthyma like presentation. Dermatol Online J 15: 10.

24. Pellaton C, Cavassini M, Jaton-Ogay K, Carron PN, Christen-Zaech S, et al (2009) Histoplasma capsulatum var. duboisii infection in a patient with AIDS Rapid diagnosis using polymerase chain reaction-sequencing. Diagn Microbiol Infect Dis 64: 85-89.

25. Ramdial PK, Calonje E (2013) Vulvovaginal infections. Pathology of the vulva and vagina. Springer-Verlag, London, pp: 13-46.

26. Sing Y, Ramdial PK, Ibrahim T (2008) Cryptococcosis masquerading as a tuboovarian abscess. Int J Gynecol Pathol 27: 37-40.

27. Blickstein I, Dgani R, Lifschitz-Mercer B (1993) Cutaneous leishmaniasis of the vulva. Int J Gynaecol Obstet 42: 46-47.

28. Weedon D (2010) Bacterial and rickettsial infections. Weedon's Skin Pathology Churchill Livingstone Elsevier, Philadelphia, pp: 548-572.

29. Maniar JK, Chitale AR, Miskeen A, Shah K, Maniar A (2005) Penicillium marneffei infection: An AIDS-defining illness. Indian J Dermatol Venereol Leprol 71: 202-204.

30. Mootsikapun P, Srikulbutr S (2006) Histoplasmosis and penicilliosis: Comparison of clinical features, laboratory findings and outcome. Int $\mathrm{J}$ Infect Dis 10: 66-71.

31. Klassen-Fischer M, McEvoy P, Neafie RC, Nelson AM (2004) Accurate diagnosis of infection with Histoplasma capsulatum var. duboisii. Clin Infect Dis 38: 595 . 